\title{
Linear Analysis of Reduced-Round CubeHash
}

\author{
Tomer Ashur and Orr Dunkelman \\ Faculty of Mathematics and Computer Science \\ Weizmann Institute of Science \\ P.O. Box 26 \\ Rehovot 76100, Israel \\ orr.dunkelman@weizmann.ac.il
}

\begin{abstract}
Recent developments in the field of cryptanalysis of hash functions has inspired NIST to announce a competition for selecting a new cryptographic hash function to join the SHA family of standards. One of the 14 second-round candidates is CubeHash designed by Daniel J. Bernstein. CubeHash is a unique hash function in the sense that it does not iterate a common compression function, and offers a structure which resembles a sponge function, even though it is not exactly a sponge function.

In this paper we analyze reduced-round variants of CubeHash where the adversary controls the full 1024-bit input to reduced-round CubeHash and can observe its full output. We show that linear approximations with high biases exist in reduced-round variants. For example, we present an 11-round linear approximation with bias of $2^{-235}$, which allows distinguishing 11round CubeHash using about $2^{470}$ queries. We also discuss the extension of this distinguisher to 12 rounds using message modification techniques. Finally, we present a linear distinguisher for 14-round CubeHash which uses about $2^{812}$ queries.
\end{abstract}

Key words: CubeHash SHA-3 competition, Linear cryptanalysis.

\section{Introduction}

Recent developments in the field of hash function cryptanalysis [1,18-20] along with new results targeted against commonly used hash functions $[6,11,26,27]$ has urged National Institute of Standards and Technology to announce a competition for the development of a new hash standard, SHA-3 [25].

The National Institute of Standards and Technology has received 64 hash function proposals for the competition, out of which 51 met the submission criteria and were accepted to the first round of the competition. Following the first round of analysis, in which the security and performance claims of the submitters were challenged, 14 candidates were selected to the second round of the SHA-3 competition. One of these 14 candidates is CubeHash designed by Daniel J. Bernstein [4].

CubeHash is a family of cryptographic hash functions, parameterized by the performance and security required. CubeHash has an internal state of 1024 bits, which are processed by calling a transformation named $T$, a tweakable number of times $r$, between introductions of new $b$-byte message blocks ( $b$ is also a tunable parameter). At the end, after a final permutation, namely, $T$ repeated $10 r$ times, $h$ bits of the state are used as an output. By selecting different values of $h, b$, and $r$, different security/performance tradeoffs are provided. Currently, several sets of 
parameters are suggested, where the "normal" security values are $r=16, b=32$ (for $h \in\{224,256,384,512\}$ ) [5]. ${ }^{1}$

In this paper we analyze the security of several variants of CubeHash against linear cryptanalysis. Our analysis found a linear approximation for 11-round Cube$\operatorname{Hash}^{2}$ with bias of $\frac{1}{4} \cdot \frac{1}{2}^{233}=2^{-235}$. We limited the analysis to biases of no less than $2^{-256}$, as we felt that a hash function offering a 512-bit security (in its strongest variant), should not be assessed with attacks taking more than $2^{512}$ queries. One can also extend the 11-round linear approximation into a 12-round distinguisher using simple message modification techniques [27] (or a chosen-plaintext linear cryptanalysis $[22])$.

We note that when removing this restriction, one can find 14-round linear approximations with bias of $2^{-406}$. Exploiting this approximation requires querying $T^{14}$ about $2^{812}$ times, which is outside the security model. At the same time, if $T$ or CubeHash are ever used in different settings, this may provide some indication concerning its security.

This paper is organized as follows: In Section 2 we describe CubeHash's compression function. In Section 3 we describe the linear approximations found for CubeHash. In Section 4 we describe how bit fixing can be used to distinguish more rounds than in the approximation. In Section 5 we quickly cover a possible application of our results. Finally, Section 6 concludes this paper.

\section{A Brief Description of CubeHash}

As mentioned before, CubeHash is a tweakable hash function, where the shared part of all its variants is the internal state (of 1024 bits), and the use of the same round function $T$.

To initialize the hash function, $h$ (the digest size), $r$ the number of times $T$ is iterated between message blocks, and $b$ the size of the message blocks (in bytes), are loaded into the state. Then, the state is updated using $10 r$ applications of $T$. At this point, the following procedure is repeated with any new message block: the $b$-byte block is XORed into the 128-byte state, and the state is updated by applying $T^{r}$ ( $r$ times applying $T$ ) to the state. After processing the padded message, the state is XORed with the constant 1 , and is processed by applying $T^{10 r}$. The output is composed of the first $h / 8$ bytes of the state.

The 1024 bits of the internal state are viewed as a sequence of 32 4-byte words $x_{00000}, x_{00001}, \ldots, x_{11111}$ each of which is interpreted in a little-endian form as a 32 bit unsigned integer. The round function $T$ of CubeHash is based on the following ten operations:

1. Add (modulo $\left.2^{32}\right) x_{0 j k l m}$ into $x_{1 j k l m}$, for all $(j, k, l, m)$.

2. Rotate $x_{0 j k l m}$ left by 7 bits, for all $(j, k, l, m)$.

3. Swap $x_{00 \mathrm{klm}}$ with $x_{01 \mathrm{klm}}$, for all $(k, l, m)$.

4. XOR $x_{1 j k l m}$ into $x_{0 j k l m}$, for all $(j, k, l, m)$.

5. Swap $x_{1 j k 0 m}$ with $x_{1 j k 1 m}$, for all $(j, k, m)$.

6. Add (modulo $\left.2^{32}\right) x_{0 j k l m}$ into $x_{1 j k l m}$, for all $(j, k, l, m)$.

\footnotetext{
${ }^{1}$ We note that there is a "formal" variant of CubeHash for which $r=16, b=1$ and $h \in\{384,512\}$.

${ }^{2}$ We note that CubeHash is a full hash function which is not easily defined in the common settings. Hence, 11-round CubeHash stands for iterating 11 times the transformation $T$. We remind the reader that our analysis usually assumes the adversary can choose the full 1024-bit input to $T$, and observe the full 1024-bit output from $T$.
} 
7. Rotate $x_{0 j k l m}$ left by 11 bits, for all $(j, k, l, m)$.

8. Swap $x_{0 j 0 l m}$ with $x_{0 j 1 l m}$, for all $(j, l, m)$.

9. XOR $x_{1 j k l m}$ into $x_{0 j k l m}$, for all $(j, k, l, m)$.

10. Swap $x_{1 j k l 0}$ with $x_{1 j k l 1}$, for all $(j, k, l)$.

The structure is represented in a little endian form, i.e., $x_{00000}$ is composed of the four least significant bytes of the state and $x_{11111}$ is composed of the most significant four. We note that the only nonlinear operations with respect to $\operatorname{GF}(2)$ are the modular additions.

\subsection{Previous Results on CubeHash}

Following its simple structure, CubeHash has received a lot of cryptanalytic attention. Some of the attacks, such as the ones of $[7,21]$, can be applied to CubeHash, independent of the actual $T$ (as long as it is invertible). These attacks target the preimage resistance of CubeHash, and exploit the fact that as all components are invertible, and as the adversary can control $b$-bytes of the internal state directly, it is possible to find a preimage in about $2^{512-4 b}$ CubeHash computations.

The second type of results, tried to analyze reduced-round variants of CubeHash for collisions. In [2], a collision for CubeHash2/120-512 is given. Collisions for CubeHash1/45 and 2/89 are given in [14], and for CubeHash4/48 and CubeHash4/64 are produced by $[9,10]$. A more general methodology to obtain such collisions is described in [8], where variants up to CubeHash7/64 are successfully analyzed.

A third type of attacks/observations concerning CubeHash deal with the symmetric structure of $T$. For example, if at the input all $x_{0 j k l m}$ words are equal, and all $x_{1 j k l m}$ words are equal (not necessarily equal to the value of the $x_{0 j k l m}$ ), then the same property holds in the output as well. The first analysis of this type of properties is given in the original submission document [4]. In [3], several additional classes of "symmetric" states are observed, and their use is analyzed. Recently, these classes were expanded to include a larger number of states (and structures) in [16].

Despite all the above-mentioned work, CubeHash is still considered secure, as no attack comes close to offer complexity which is significantly better than generic attacks. ${ }^{3}$ To the best of our knowledge this work is the first one that succeeds to offer some non-trivial property of more than 10 rounds of $T$.

\section{Linear Approximation of CubeHash}

Linear cryptanalysis [24] is a useful cryptanalytic tool in the world of block cipher cryptanalysis. The cryptosystem is linearly approximated (by an expression that holds with some bias), and the adversary gains information concerning the key, by observing sufficient amount of plaintext/ciphertext pairs satisfying the approximation.

In the context of hash functions, linear cryptanalysis has received very little attention, unlike differential cryptanalysis. The reason for that seems that while differential cryptanalysis can be directly used to offer collisions or preimages, linear cryptanalysis seems to be restricted to very rare cases (i.e., where the bias is extremely high).

\footnotetext{
${ }^{3}$ We note that while the preimage attacks of $[7,21]$ may offer a small speed-up with respect to generic attacks, their memoryless variants are not much faster than exhaustive search. Moreover, as the submission document lists this as a known issue, this flaw is not considered too harmful by many.
} 
At the same time, the use of linear approximation to assess the security of a hash function can shed some light on whether the underlying components offer the required security. Moreover, linear approximations of the compression function might be useful when discussing MACs built on top of the hash function (suggesting a detectable linear bias in the output).

\subsection{Linear Approximation of Addition Modulo $2^{32}$}

CubeHash uses a mixture of XORs, rotations, and additions. While the first two can be easily handled in the linear cryptanalysis framework, the approximation of the modular addition possess several problems, mostly due to the carry chains.

One of the papers studying the cryptographic properties of modular addition is [12] which studies the carry effects on linear approximations. In the paper, Cho and Pieperzyk show that approximating two consecutive bits can overcome some of the inherent problems of carry chains. Namely, if $\lambda$ is a mask of two consecutive bits (in any position) then: $\lambda \cdot(x+y)=\lambda(x \oplus y)$ with probability $3 / 4$ (i.e., a bias of $1 / 4)$.

We analyzed several cases where $\lambda$ contains pairs of consecutive bits, e.g., two pairs of consecutive pairs, and even when these pairs appear immediately after each other (i.e., $\lambda$ is composed of four consecutive bits set to 1). Our analysis shows that with respect to linear cryptanalysis, these pairs can be treated as two separate independent instances. For example, the probability that $\lambda \cdot(x+y)=\lambda(x \oplus y)$ for $\lambda$ whose four most significant bits are 1 , while the rest are 0 , is $10 / 16$ (suggesting the expected bias of $\left.2 \cdot(1 / 4)^{2}=1 / 8\right)$.

\subsection{The Linear Approximation of the Round Function of CubeHash}

Our first attempt in understanding the security of CubeHash against linear cryptanalysis was a very simple experiment. We looked at all possible masks which had only one pair of two consecutive bits active, and tried to extend this mask as many rounds as possible in the forward direction. At some point, the resulting mask had a divided pair of bits, i.e., a pair of bits that due to the rotations used in CubeHash were sent one to the LSB of a word, and one to the MSB of a word. Such a mask does no longer fall under the type of masks considered in [12], and our experiments show that such a mask has a very low bias when considering addition.

After performing the search in the forward direction, we repeated the experiment, this time running the light mask in the backward direction (i.e., through $T^{-1}$ ) as many rounds as possible. The results obtained in these experiments are shown in Tables 1 and 2, which present the number of possible linear approximations of that form in the forward and the backward directions (along with the associated bias). The longest of which covers 10 rounds in any direction.

Following the surprisingly long approximations, we decided to explore pairs of pairs (i.e., four active bits in the starting mask), repeating the process of analyzing the forward direction as well as the backward direction. These results are summarized in Tables 3 and 4.

We also combined the forward and the backward approximations to form a series of approximations for as many rounds as could, using the combination of this type of approximations. In Table 5 we offer input/output masks of the best approximations we found.

Following the fact that CubeHash aims to offer at most a $2^{512}$ security, we decided to concentrate at approximations of bias up to $2^{-256}$ (as detecting smaller 
Table 1. Number of Linear Approximations Following the Consecutive Masks Approach (Starting from a Mask with One Consecutive Pair in the Forward Direction)

\begin{tabular}{|c|c|c|}
\hline $\mid$ Rounds & Bias & $\mid$ Number of Approximations \\
\hline 1 & $\frac{1}{4} \cdot \frac{1}{2}^{0}=2^{-2}$ & 480 \\
\hline 1 & $\frac{1}{4} \cdot \frac{1}{2}^{1}=2^{-3}$ & 16 \\
\hline 1 & $\frac{1}{4} \cdot \frac{1}{2}^{2}=2^{-4}$ & 480 \\
\hline 1 & $\frac{1}{4} \cdot \frac{1}{2}^{3}=2^{-5}$ & 16 \\
\hline 2 & $\frac{1}{4} \cdot \frac{1}{2}^{11}=2^{-13}$ & 432 \\
\hline 2 & $\frac{1}{4}^{1} \cdot \frac{1}{2}^{12}=2^{-14}$ & 16 \\
\hline 2 & $\frac{1}{4} \cdot \frac{1}{2}^{15}=2^{-17}$ & 16 \\
\hline 2 & $\frac{1}{4} \cdot \frac{1}{2}^{16}=2^{-18}$ & 416 \\
\hline 2 & $\frac{1}{4}^{1} \cdot \frac{1}{2}^{17}=2^{-19}$ & 16 \\
\hline 2 & $\frac{1}{4} \cdot \frac{1}{2}^{20}=2^{-22}$ & 16 \\
\hline 3 & $\frac{1}{4} \cdot \frac{1}{2}^{29}=2^{-31}$ & 384 \\
\hline 3 & $\frac{1}{4}^{4} \cdot \frac{1}{2}^{30}=2^{-32}$ & 16 \\
\hline 3 & $\frac{1}{4} \cdot \frac{1}{2}^{33}=2^{-35}$ & 16 \\
\hline 3 & $\frac{1}{4}^{4} \cdot \frac{1}{2}^{35}=2^{-37}$ & 352 \\
\hline 3 & $\frac{1}{4} \cdot \frac{1}{2}^{36}=2^{-38}$ & 16 \\
\hline 3 & $\frac{1}{4} \cdot \frac{1}{2}^{239}=2^{-41}$ & 16 \\
\hline 4 & $\frac{1}{4} \cdot \frac{1}{2}^{66}=2^{-68}$ & 336 \\
\hline 4 & $\frac{1}{4} \cdot \frac{1}{2}^{67}=2^{-69}$ & 16 \\
\hline 4 & $\frac{1}{4} \cdot \frac{1}{2}^{70}=2^{-72}$ & 16 \\
\hline 4 & $\frac{1}{4} \cdot \frac{1}{2}^{74}=2^{-76}$ & 288 \\
\hline 4 & $\frac{1}{4} \cdot \frac{1}{2}^{75}=2^{-77}$ & 16 \\
\hline 4 & $\frac{1}{4} \cdot \frac{1}{2}^{78}=2^{-80}$ & 16 \\
\hline 5 & $\frac{1}{4} \cdot \frac{1}{2}^{113}=2^{-115}$ & 272 \\
\hline 5 & $\frac{1}{4} \cdot \frac{1}{2}^{114}=2^{-116}$ & 240 \\
\hline 5 & $\frac{4}{4} \cdot \frac{1}{2}^{115}=2^{-117}$ & 16 \\
\hline 5 & $\frac{1}{4} \cdot \frac{1}{2}{ }^{117}=2^{-119}$ & 16 \\
\hline 5 & $\left|\frac{1}{4} \cdot \frac{1}{2}^{118}=2^{-120}\right|$ & 16 \\
\hline 6 & $\frac{1}{4} \cdot \frac{1}{2}^{169}=2^{-171}$ & 208 \\
\hline 6 & $\frac{4}{4} \cdot \frac{1}{2}^{170}=2^{-172}$ & 16 \\
\hline 6 & $\frac{4}{4} \cdot \frac{1}{2}^{171}=2^{-173}$ & 160 \\
\hline 6 & $\frac{4}{4} \cdot \frac{1}{2} 172=2^{-174}$ & 16 \\
\hline 6 & $\frac{1}{4} \cdot \frac{1}{2}^{2} 173=2^{-175}$ & 16 \\
\hline 6 & $\frac{1}{4} \cdot \frac{1}{2}^{175}=2^{-177} \mid$ & 16 \\
\hline 7 & $\frac{1}{4} \cdot \frac{1}{2}^{236}=2^{-238}$ & 96 \\
\hline 7 & $\frac{1}{4} \cdot \frac{1}{2} 237=2^{-239}$ & 16 \\
\hline 7 & $\frac{1}{4} \cdot \frac{1}{2}^{238}=2^{-240}$ & 144 \\
\hline 7 & $\frac{1}{4} \cdot \frac{1}{2}^{239}=2^{-241}$ & 16 \\
\hline 7 & $\frac{1}{4} \cdot \frac{1}{2}{ }^{240}=2^{-242}$ & 16 \\
\hline 7 & $\left|\frac{1}{4} \cdot \frac{1}{2}{ }^{242}=2^{-244}\right|$ & 16 \\
\hline 8 & $\frac{1}{4} \cdot \frac{1}{2}^{346}=2^{-348}$ & 32 \\
\hline 8 & $\frac{1}{4} \cdot \frac{1}{2} 347=2^{-349}$ & 16 \\
\hline 8 & $\frac{4}{4} \cdot \frac{1}{2}^{2350}=2^{-352}$ & 16 \\
\hline 8 & $\frac{1}{4} \cdot \frac{1}{2} 353=2^{-355}$ & 80 \\
\hline 8 & $\frac{1}{4} \cdot \frac{1}{2}^{2354}=2^{-356}$ & 16 \\
\hline 8 & $\frac{1}{4} \cdot \frac{1}{2} 357=2^{-359}$ & 16 \\
\hline 9 & $\frac{1}{4} \cdot \frac{1}{2}{ }^{445}=2^{-447}$ & 16 \\
\hline 9 & $\frac{4}{4} \cdot \frac{1}{2}{ }^{481}=2^{-483}$ & 32 \\
\hline 9 & $\frac{1}{4} \cdot \frac{1}{2}{ }^{485}=2^{-487}$ & 16 \\
\hline 10 & $\mid \frac{1}{4} \cdot \frac{1}{2}^{550}=2^{-552}$ & 16 \\
\hline
\end{tabular}

biases requires more than $2^{512}$ queries). The longest possible approximation which adheres to this restriction is of 11 rounds and has a bias of $2^{-235}$ which is fully described in Table 6. 
Table 2. Number of Linear Approximations Following the Consecutive Masks Approach (Starting from a Mask with One Consecutive Pair in the Backward Direction)

\begin{tabular}{|c|c|c|}
\hline Rounds & Bias & |Number of Approximations \\
\hline 1 & $\frac{1}{4} \cdot \frac{1}{2}^{2}=2^{-4}$ & 496 \\
\hline 1 & $\frac{1}{4} \cdot \frac{1}{2}^{3}=2^{-5}$ & 480 \\
\hline 1 & $\frac{1^{4}}{4} \cdot \frac{1}{2}^{4}=2^{-6}$ & 16 \\
\hline 2 & $\frac{1}{4} \cdot \frac{1}{2}^{12}=2^{-14}$ & 448 \\
\hline 2 & $\frac{1}{4} \cdot \frac{1}{2}^{13}=2^{-15}$ & 16 \\
\hline 2 & $\frac{1}{4}_{4} \cdot \frac{1}{2}^{14}=2^{-16}$ & 16 \\
\hline 2 & $\frac{1}{4}^{1} \cdot \frac{1}{2}^{18}=2^{-20}$ & 416 \\
\hline 2 & $\frac{1}{4} \cdot \frac{1}{2}^{19}=2^{-21}$ & 32 \\
\hline 3 & $\frac{1}{4} \cdot \frac{1}{2}^{29}=2^{-31}$ & 368 \\
\hline 3 & $\frac{1}{4}^{4} \cdot \frac{1}{2}^{30}=2^{-32}$ & 32 \\
\hline 3 & $\frac{1}{4} \cdot \frac{1}{2}^{31}=2^{-33}$ & 16 \\
\hline 3 & $\frac{1}{4} \cdot \frac{1}{2}^{41}=2^{-43}$ & 336 \\
\hline 3 & $\frac{1}{4}^{1} \cdot \frac{1}{2}^{42}=2^{-44}$ & 48 \\
\hline 4 & $\frac{1}{4}^{1} \frac{1}{2}^{60}=2^{-62}$ & 304 \\
\hline 4 & $\frac{1}{4}^{1} \cdot \frac{1}{2}^{61}=2^{-63}$ & 16 \\
\hline 4 & $\frac{1}{4} \cdot \frac{1}{2}^{62}=2^{-64}$ & 32 \\
\hline 4 & $\frac{1}{4} \cdot \frac{1}{2}^{85}=2^{-87}$ & 256 \\
\hline 4 & $\frac{1}{4} \cdot \frac{1}{2}^{86}=2^{-88}$ & 48 \\
\hline 5 & $\mid \frac{1}{4} \cdot \frac{1}{2}^{102}=2^{-104}$ & 240 \\
\hline 5 & $\frac{1}{4} \cdot \frac{1}{2}^{103}=2^{-105}$ & 32 \\
\hline 5 & $\frac{1}{4} \cdot \frac{1}{2}{ }^{104}=2^{-106}$ & 16 \\
\hline 5 & $\frac{1}{4} \cdot \frac{1}{2}^{134}=2^{-136}$ & 224 \\
\hline 5 & $\frac{1}{4} \cdot \frac{1^{1}}{2}{ }^{135}=2^{-137}$ & 16 \\
\hline 6 & $\mid \frac{1}{4} \cdot \frac{1}{2}^{149}=2^{-151}$ & 192 \\
\hline 6 & $\frac{1}{4} \cdot \frac{1}{2}^{150}=2^{-152}$ & 16 \\
\hline 6 & $\frac{1}{4} \cdot \frac{1}{2}{ }^{151}=2^{-153}$ & 16 \\
\hline 6 & $\frac{1}{4} \cdot \frac{1}{2}{ }^{197}=2^{-199}$ & 144 \\
\hline 6 & $\mid \frac{1}{4} \cdot \frac{1}{2}{ }^{198}=2^{-200}$ & 48 \\
\hline 7 & $\frac{1}{4} \cdot \frac{1}{2}^{212}=2^{-214}$ & 112 \\
\hline 7 & $\frac{1}{4} \cdot \frac{1}{2}{ }^{213}=2^{-215}$ & 32 \\
\hline 7 & $\frac{1}{4} \cdot \frac{1}{2}{ }^{214}=2^{-216}$ & 16 \\
\hline 7 & $\frac{1}{4} \cdot \frac{1}{2}{ }^{277}=2^{-279}$ & 80 \\
\hline 7 & $\mid \frac{1}{4} \cdot \frac{1}{2}^{278}=2^{-280}$ & 32 \\
\hline 8 & $\mid \frac{1}{4} \cdot \frac{1}{2}^{308}=2^{-310}$ & 48 \\
\hline 8 & $\frac{1}{4} \cdot \frac{1}{2}{ }^{309}=2^{-311}$ & 16 \\
\hline 8 & $\frac{1}{4} \cdot \frac{1}{2} 310=2^{-312}$ & 32 \\
\hline 8 & $\frac{1}{4} \cdot \frac{1}{2}{ }^{407}=2^{-409}$ & 48 \\
\hline 8 & $\left|\frac{1}{4} \cdot \frac{1}{2}{ }^{409}=2^{-411}\right|$ & 16 \\
\hline 9 & $\left|\frac{1}{4} \cdot \frac{1}{2}^{418}=2^{-420}\right|$ & 32 \\
\hline 10 & $\mid \frac{1}{4} \cdot \frac{1}{2}{ }^{477}=2^{-479}$ & 16 \\
\hline
\end{tabular}

For those interested in assessing the full security that might be offered by the 1024-bit transformation $T$, we note that there also exists a 14-round linear approximation with a bias of $2^{-406}$. We outline the full 14 -round approximation in Tables 7 and 8 .

\section{Message Modification Techniques — A Chosen-Plaintext Linear Approximations}

Linear cryptanalysis relies on collecting a large number of input/output pairs, and verifying whether they satisfy the approximation or not. In [22] Knudsen and Math- 
Table 3. Number of Approximations with a Given Bias Starting from a Pair of Pair of Active Bits (Forward Direction)

\begin{tabular}{|c|c|c|}
\hline Rounds & Bias & |Number of Approximations \\
\hline $\begin{array}{l}1 \\
1 \\
1\end{array}$ & $\begin{array}{l}\frac{1}{4} \cdot \frac{1}{2}^{1}=2^{-3} \\
\frac{1}{4} \cdot \frac{1}{2}^{3}=2^{-5} \\
\frac{1}{4} \cdot \frac{1}{2}^{5}=2^{-7}\end{array}$ & $\begin{array}{l}115472 \\
228128 \\
113152\end{array}$ \\
\hline $\begin{array}{l}2 \\
2 \\
2 \\
2\end{array}$ & 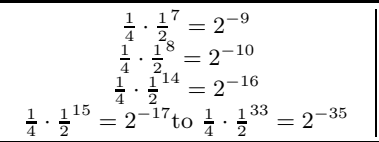 & $\begin{array}{c}232 \\
448 \\
848 \\
301480\end{array}$ \\
\hline $\begin{array}{l}3 \\
3 \\
3 \\
3 \\
\end{array}$ & $\begin{aligned} & \frac{1}{4} \cdot \frac{1}{2}^{23}=2^{-25} \\
& \frac{1}{4} \cdot \frac{1}{2}^{25}=2^{-27} \\
& \frac{1}{4} \cdot \frac{1}{2}^{35}=2^{-37} \\
& \frac{1}{4} \cdot \frac{1}{2}^{37}=2^{-39} \text { to } \frac{1}{4} \cdot \frac{1}{2}^{71}=2^{-73}\end{aligned}$ & $\begin{array}{c}208 \\
384 \\
352 \\
188144 \\
\end{array}$ \\
\hline $\begin{array}{l}4 \\
4 \\
4 \\
4\end{array}$ & $\begin{aligned} \frac{1}{4} \cdot \frac{1}{2}{ }^{45}=2^{-47} \\
\frac{1}{4} \cdot \frac{1}{2}^{53}=2^{-55} \\
\frac{1}{4} \cdot \frac{1}{2}{ }^{73}=2^{-75} \\
\frac{1}{4} \cdot \frac{1}{2}^{77}=2^{-79} \text { to } \frac{1}{4} \cdot \frac{1}{2}^{149}=2^{-151}\end{aligned}$ & $\begin{array}{c}184 \\
320 \\
304 \\
98288 \\
\end{array}$ \\
\hline $\begin{array}{l}5 \\
5 \\
5 \\
5\end{array}$ & $\begin{array}{c}\frac{1}{4} \cdot \frac{1}{2} \frac{1}{2}^{87}=2^{-89} \\
\frac{1}{4} \cdot \frac{1}{2}=2^{-96} \\
\frac{1}{4} \cdot \frac{1^{2}}{2}{ }^{121}=2^{-123} \\
\frac{1}{4} \cdot \frac{1}{2}^{122}=2^{-124} \text { to } \frac{1}{4} \cdot \frac{1}{2}^{229}=2^{-231}\end{array}$ & $\begin{array}{c}160 \\
256 \\
128 \\
61056 \\
\end{array}$ \\
\hline $\begin{array}{l}6 \\
6 \\
6 \\
6 \\
\end{array}$ & $\begin{aligned} & \frac{1}{4} \cdot \frac{1}{2}^{123}=2^{-125} \\
& \frac{1}{4} \cdot \frac{1}{2}^{139}=2^{-141} \\
& \frac{1}{4} \cdot \frac{1}{2}^{179}=2^{-181} \\
& \frac{1}{4} \cdot \frac{1}{2}^{185}=2^{-187} \text { to } \frac{1}{4} \cdot \frac{1}{2}^{343}=2^{-345}\end{aligned}$ & $\begin{array}{c}128 \\
192 \\
272 \\
33632 \\
\end{array}$ \\
\hline \begin{tabular}{l|l|}
7 & \\
7 & \\
7 & \\
7 & 2 \\
\end{tabular} & $\begin{aligned} \frac{1}{4} \cdot \frac{1}{2}^{181} & =2^{-183} \\
\frac{1}{4} \cdot \frac{1}{2}^{201} & =2^{-203} \\
\frac{1}{4} \cdot \frac{1}{2}^{249} & =2^{-251} \\
\frac{1}{4} \cdot \frac{1}{2}^{257}=2^{-259} & \text { to } \frac{1}{4} \cdot \frac{1}{2}^{477}=2^{-479}\end{aligned}$ & $\begin{array}{c}96 \\
128 \\
64 \\
14256 \\
\end{array}$ \\
\hline \begin{tabular}{l|r|}
8 & \\
8 & \\
8 & \\
8 & \\
\end{tabular} & $\begin{aligned} \frac{1}{4} \cdot \frac{1}{2}^{251} & =2^{-253} \\
\frac{1}{4} \cdot \frac{1}{2}^{288} & =2^{-290} \\
\frac{1}{4} \cdot \frac{1}{2} & =2^{-370} \\
\frac{1}{4} \cdot \frac{1}{2}^{369}=2^{-371} & \text { to } \frac{1}{4} \cdot \frac{1}{2}^{693}=2^{-695}\end{aligned}$ & $\begin{array}{c}64 \\
64 \\
48 \\
3120 \\
\end{array}$ \\
\hline \begin{tabular}{l|l|}
9 & \\
9 & \\
9 & \\
9 &
\end{tabular} & 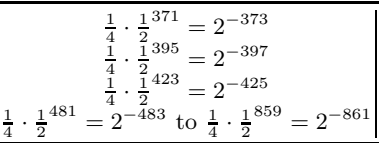 & $\begin{array}{c}32 \\
16 \\
16 \\
336\end{array}$ \\
\hline \begin{tabular}{l|}
10 \\
10 \\
10 \\
10
\end{tabular} & $\begin{aligned} \frac{1}{4} \cdot \frac{1}{2}^{425} & =2^{-427} \\
\frac{1}{4} \cdot \frac{1}{2} 571 & =2^{-573} \\
& \frac{1}{4} \cdot \frac{1}{2} 597 \\
= & 2^{-599} \\
\frac{1}{4} \cdot \frac{1}{2}^{697}= & 2^{-699} \text { to } \frac{1}{4} \cdot \frac{1}{2}^{993}=2^{-995}\end{aligned}$ & $\begin{array}{l}16 \\
32 \\
16 \\
48 \\
\end{array}$ \\
\hline $\begin{array}{l}11 \\
11 \\
\end{array}$ & $\begin{array}{l}\frac{1}{4} \cdot \frac{1}{2}^{620}=2^{-622} \\
\frac{1}{4} \cdot \frac{1}{2}{ }^{663}=2^{-665}\end{array}$ & $\begin{array}{l}32 \\
16 \\
\end{array}$ \\
\hline 12 & $\frac{1}{4} \cdot \frac{1}{2}^{681}=2^{-683}$ & 32 \\
\hline 13 & $\frac{1}{4} \cdot \frac{1}{2}^{737}=2^{-739}$ & 32 \\
\hline 14 & $\frac{1}{4} \cdot \frac{1}{2}^{786}=2^{-788}$ & 32 \\
\hline 15 & $\frac{1}{4} \cdot \frac{1}{2}^{855}=2^{-857}$ & 32 \\
\hline 16 & $\frac{1}{4} \cdot \frac{1}{2}^{983}=2^{-985}$ & 32 \\
\hline
\end{tabular}

iassen show that there are cases in which one can "help" the linear approximation to be satisfied by properly selecting the inputs. 
Table 4. Number of Approximations with a Given Bias Starting from a Pair of Pair of Active Bits (Backward Direction)

\begin{tabular}{|c|c|c|}
\hline $\mid \overline{\text { Rounds }}$ & Bias & $\mid$ Number of Approximations \\
\hline $\begin{array}{l}1 \\
1 \\
1 \\
1\end{array}$ & $\begin{array}{c}\frac{1}{4} \cdot \frac{1}{2}^{0}=2^{-2} \\
\frac{1}{4} \cdot \frac{1}{2}^{1}=2^{-3} \\
\frac{1}{4} \cdot \frac{1}{2}^{2}=2^{-4} \\
\frac{1}{4} \cdot \frac{1}{2}^{3}=2^{-5} \text { to } \frac{1}{4} \cdot \frac{1}{2}^{7}=2^{-9}\end{array}$ & $\begin{array}{c}464 \\
240 \\
448 \\
411040\end{array}$ \\
\hline $\begin{array}{l}2 \\
2 \\
2 \\
2\end{array}$ & $\begin{aligned} \frac{1}{4} \cdot \frac{1}{2}^{5} & =2^{-7} \\
\frac{1}{4}^{4} \cdot \frac{1}{2}^{11} & =2^{-13} \\
\frac{1}{4} \cdot \frac{1}{2}^{13} & =2^{-15} \\
\frac{1}{4} \cdot \frac{1}{2}^{17}=2^{-19} & \text { to } \frac{1}{4} \cdot \frac{1}{2}^{37}=2^{-39}\end{aligned}$ & $\begin{array}{c}216 \\
400 \\
368 \\
250224\end{array}$ \\
\hline $\begin{array}{l}3 \\
3 \\
3 \\
3 \\
3 \\
3\end{array}$ & $\begin{aligned} \frac{1}{4} \cdot \frac{1}{2}^{19} & =2^{-21} \\
\frac{1}{4} \cdot \frac{1}{2} 29 & =2^{-31} \\
\frac{1}{4} \cdot \frac{1}{2} 31 & =2^{-33} \\
\frac{1}{4} \cdot \frac{1}{2} 35 & =2^{-37} \\
\frac{1}{4} \cdot \frac{1}{2} & =2^{-41} \text { to } \frac{1}{4} \cdot \frac{1}{2}^{83}=2^{-85}\end{aligned}$ & $\begin{array}{c}184 \\
352 \\
304 \\
152 \\
136544\end{array}$ \\
\hline $\begin{array}{l}4 \\
4 \\
4 \\
4 \\
4\end{array}$ & $\begin{aligned} \frac{1}{4} \cdot \frac{1}{2}{ }^{37}=2^{-39} \\
\frac{1}{4} \cdot \frac{1}{2} 66=2^{-68} \\
\frac{1}{4} \cdot \frac{1}{2} 75=2^{-77} \\
\frac{1}{4} \cdot \frac{1}{2} 77=2^{-79} \\
{\frac{1}{4} \cdot \frac{1}{2}^{80}=}^{-2^{-82}} \text { to } \frac{1}{4} \cdot \frac{1}{2}^{172}=2^{-174}\end{aligned}$ & $\begin{array}{c}152 \\
528 \\
120 \\
144 \\
69664 \\
\end{array}$ \\
\hline $\begin{array}{l}5 \\
5 \\
5 \\
5 \\
5 \\
\end{array}$ & 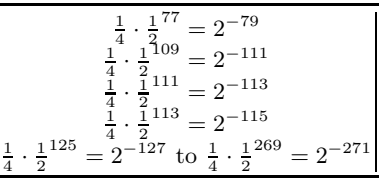 & $\begin{array}{c}120 \\
96 \\
192 \\
240 \\
43344 \\
\end{array}$ \\
\hline $\begin{array}{l}6 \\
6 \\
6 \\
6 \\
6\end{array}$ & $\begin{aligned} \frac{1}{4} \cdot \frac{1}{2}^{111} & =2^{-113} \\
\frac{1}{4} \cdot \frac{1}{2} 163 & =2^{-165} \\
\frac{1}{4} \cdot \frac{1}{2} & 169 \\
\frac{1}{4} \cdot \frac{1}{2} & =2^{-179}=2^{-181} \\
\frac{1}{4} \cdot \frac{1}{2}^{187}=2^{-189} & \text { to } \frac{1}{4} \cdot \frac{1}{2}^{387}=2^{-389}\end{aligned}$ & $\begin{array}{c}96 \\
168 \\
176 \\
112 \\
18672\end{array}$ \\
\hline $\begin{array}{l}7 \\
7 \\
7 \\
7 \\
7 \\
\end{array}$ & 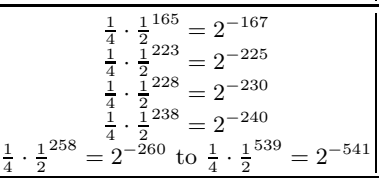 & $\begin{array}{c}56 \\
24 \\
64 \\
112 \\
5904 \\
\end{array}$ \\
\hline $\begin{array}{l}8 \\
8 \\
8 \\
8\end{array}$ & 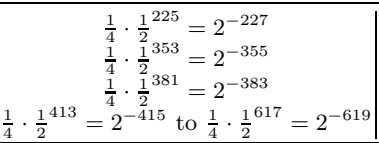 & $\begin{array}{c}24 \\
32 \\
16 \\
272 \\
\end{array}$ \\
\hline $\begin{array}{l}9 \\
9 \\
9\end{array}$ & $\begin{array}{l}\frac{1}{4} \cdot 1^{481}=2^{-483} \\
\frac{1}{4} \cdot \frac{1}{2} 2^{527}=2^{-529} \\
\frac{1}{4} \cdot \frac{1}{2}{ }^{679}=2^{-681}\end{array}$ & $\begin{array}{l}32 \\
16 \\
32\end{array}$ \\
\hline $\begin{array}{l}10 \\
10\end{array}$ & $\begin{array}{l}\frac{1}{4} \cdot \frac{1}{2}^{550}=2^{-552} \\
\frac{1}{4} \cdot \frac{1}{2}{ }^{773}=2^{-775}\end{array}$ & $\begin{array}{l}32 \\
16\end{array}$ \\
\hline $\begin{array}{l}11 \\
11\end{array}$ & $\begin{array}{l}\frac{1}{4} \cdot \frac{1}{2}{ }^{599}=2^{-601} \\
\frac{1}{4} \cdot \frac{1}{2} 863=2^{-865}\end{array}$ & $\begin{array}{l}16 \\
16\end{array}$ \\
\hline 12 & $\frac{1}{4} \cdot \frac{1}{2}^{953}=2^{-955}$ & 16 \\
\hline
\end{tabular}

In the case of modular addition, the linear approximation which we use is satisfied whenever one of the LSBs of the approximated bits is 0 . This allows preselecting inputs for which the approximation holds with probability 1.

When considering an extension of the linear approximation shown in Table 6 by calculating it one round backward as described in Table 9 , we can fix 80 input bits to zero, thus ensuring that the approximation holds for the first layer of additions with probability 1 . These 80 bits are the ones masked by $x_{10001}=00080888_{x}, x_{10011}=$ 
Table 5. A trade-off of biases and rounds. Each line shows the best bias in this setting

\begin{tabular}{|c|c|c|c|}
\hline Rounds & Input mask & Output mask & Bias \\
\hline 7 & $\begin{array}{l}x_{00001}=06001806, x_{00011}=06001806 \\
x_{00101}=00 c 03030, x_{00111}=00 c 03030 \\
x_{01001}=000 c 0303, x_{01011}=000 c 0303 \\
x_{10100}=00000030, x_{10110}=00000030 \\
x_{11001}=000 c 0303, x_{11011}=000 c 0303\end{array}$ & $\begin{array}{l}x_{00000}=00180606, x_{00010}=00180606 \\
x_{01101}=00000060, x_{01111}=00000060 \\
x_{10001}=00180606, x_{10011}=00180606 \\
x_{10101}=c 0 c 00300, x_{10111}=c 0 c 00300 \\
x_{11101}=60018060, x_{11111}=60018060\end{array}$ & $\frac{1}{4} \cdot \frac{1}{2}^{81}=2^{-83}$ \\
\hline 8 & $\begin{array}{l}x_{00000}=06001806, x_{00010}=06001806 \\
x_{01101}=66600060, x_{01111}=66600060 \\
x_{10001}=06001806, x_{10011}=06001806 \\
x_{10101}=00 c 0 c 003, x_{10111}=00 c 0 c 003 \\
x_{11101}=60600180, x_{11111}=60600180\end{array}$ & $\begin{array}{l}x_{00000}=00180606, x_{00010}=00180606 \\
x_{01101}=00000060, x_{01111}=00000060 \\
x_{10001}=00180606, x_{10011}=00180606 \\
x_{10101}=c 0 c 00300, x_{10111}=c 0 c 00300 \\
x_{11101}=60018060, x_{11111}=60018060\end{array}$ & $\frac{1}{4} \cdot \frac{1}{2}^{121}=2^{-123}$ \\
\hline 9 & $\begin{array}{c}x_{00001}=00181998, x_{00011}=00181998 \\
x_{00101}=c 0 c c c 000, x_{00111}=c 0 c c c 000 \\
x_{01001}=0 c 0 c c c 00, x_{01011}=0 c 0 c c c 00 \\
x_{10100}=00 c 0 c 003, x_{10110}=00 c 0 c 003 \\
x_{11001}=0 c 0 c c c 00, x_{11011}=0 c 0 c c c 00\end{array}$ & $\begin{array}{l}x_{00000}=00180606, x_{00010}=00180606 \\
x_{01101}=00000060, x_{01111}=00000060 \\
x_{10001}=00180606, x_{10011}=00180606 \\
x_{10101}=c 0 c 00300, x_{10111}=c 0 c 00300 \\
x_{11101}=60018060, x_{11111}=60018060\end{array}$ & $\frac{1}{4} \cdot \frac{1}{2}^{155}=2^{-157}$ \\
\hline 10 & $\begin{array}{c}x_{00001}=00181998, x_{00011}=00181998 \\
x_{00101}=c 0 c c c 000, x_{00111}=c 0 c c c 000 \\
x_{01001}=0 c 0 c c c 00, x_{01011}=0 c 0 c c c 00 \\
x_{10100}=00 c 0 c 003, x_{10110}=00 c 0 c 003 \\
x_{11001}=0 c 0 c c c 00, x_{11011}=0 c 0 c c c 00\end{array}$ & 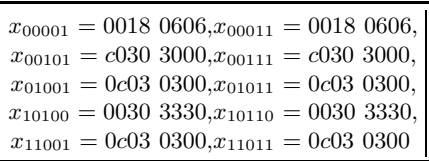 & $\frac{1}{4} \cdot \frac{1}{2}^{197}=2^{-199}$ \\
\hline 11 & $\begin{array}{c}x_{00001}=00181998, x_{00011}=00181998 \\
x_{00101}=c 0 c c c 000, x_{00111}=c 0 c c c 000 \\
x_{01001}=0 c 0 c c c 00, x_{01011}=0 c 0 c c c 00 \\
x_{10100}=00 c 0 c 003, x_{10110}=00 c 0 c 003 \\
x_{11001}=0 c 0 c c c 00, x_{11011}=0 c 0 c c c 00\end{array}$ & $\begin{array}{l}x_{00000}=81998001, x_{00010}=81998001 \\
x_{01101}=18180060, x_{01111}=18180060 \\
x_{10001}=81998001, x_{10011}=81998001 \\
x_{10101}=00303330, x_{10111}=00303330 \\
x_{11101}=18199800, x_{11111}=18199800\end{array}$ & $\frac{1}{4} \cdot \frac{1}{2}^{233}=2^{-235}$ \\
\hline 12 & $\begin{array}{l}x_{00000}=18199800, x_{00010}=18199800 \\
x_{01101}=e 7999 f 81, x_{01111}=e 7999 f 81 \\
x_{10001}=18199800, x_{10011}=18199800 \\
x_{10101}=00030333, x_{10111}=00030333 \\
x_{11101}=01819980, x_{11111}=01819980\end{array}$ & $\begin{array}{l}x_{00000}=99800181, x_{00010}=99800181 \\
x_{01101}=18006018, x_{01111}=18006018 \\
x_{10001}=99800181, x_{10011}=99800181 \\
x_{10101}=30333000, x_{10111}=30333000 \\
x_{11101}=19980018, x_{11111}=19980018\end{array}$ & $\frac{1}{4} \cdot \frac{1}{2}^{287}=2^{-289}$ \\
\hline 13 & $\begin{array}{c}x_{00000}=06660006, x_{00010}=06660006 \\
x_{01101}=e 667 \text { e079, } x_{01111}=e 667 \text { e079 } \\
x_{10001}=06660006, x_{10011}=06660006 \\
x_{10101}=00 c 0 c c c 0, x_{10111}=00 c 0 \text { ccc0 } \\
x_{11101}=60666000, x_{11111}=60666000\end{array}$ & 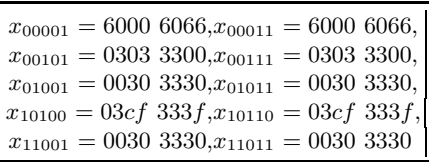 & $\frac{1}{4} \cdot \frac{1}{2}^{345}=2^{-347}$ \\
\hline 14 & $\begin{array}{l}x_{00001}=3 c c c f c 0 f, x_{00011}=3 c c c f c 0 f \\
x_{00101}=67 e 079 e 6, x_{00111}=67 e 079 e 6 \\
x_{01001}=667 e 079 e, x_{01011}=667 e 079 e \\
x_{10100}=66600060, x_{10110}=66600060 \\
x_{11001}=667 e 079 e, x_{11011}=667 e 079 e\end{array}$ & $\begin{array}{l}x_{00001}=30333000, x_{00011}=30333000 \\
x_{00101}=99800181, x_{00111}=99800181 \\
x_{01001}=19980018, x_{01011}=19980018 \\
x_{10100}=999 f \quad 81 e 7, x_{10110}=999 f 81 e 7 \\
x_{11001}=19980018, x_{11011}=19980018\end{array}$ & $\frac{1}{4}^{1} \cdot \frac{1}{2}^{405}=$ \\
\hline
\end{tabular}

$00080888_{x}, x_{10101}=11000101_{x}, x_{10111}=11000101_{x}$ and the whole words $x_{11101}$ and $x_{11111}$. We note that one can pick other sets of bits (where any fixed bit from $x_{0 j k l m}$ can be exchanged for a bit in $\left.x_{1 j k l m}\right)$.

Fixing bits for the next layer is a bit more tricky, as it requires to fix some internal state bit (after an XOR or addition) is 0 . This task is a bit harder due to carry issues. More precisely, to fix bit $i$ of $x_{1 j k l m}$ after the first five operations of $T$, it is required that bit $i$ of $x_{1 j k \overline{l m}}$ is 0 after the first operation of $T$. This specific bit depends on the corresponding carry chain.

A simple solution would be to fix one of the words $x_{0 j k \bar{l} m}$ or $x_{1 j k \bar{l} m}$ to zero, ensuring no carrys are produced during the addition $x_{1 j k \bar{l} m} \leftarrow x_{0 j k \bar{l} m}+x_{1 j k \bar{l} m}$. By additionally fixing bit $i$ of both $x_{0 j k \bar{l} m}$ and $x_{1 j k \bar{l} m}$ to zero, we can guarantee that the bit that enters the second layer of additions is indeed zero. 
Table 6. The 11-round linear approximation with bias $\frac{1}{4} \cdot \frac{1}{2}^{233}=2^{-235}$

\begin{tabular}{|c|c|c|c|}
\hline Round & $\begin{array}{c}\text { Mask } \\
\text { (before the round) }\end{array}$ & Bias & $\begin{array}{c}\text { Hamming } \\
\text { Weight }\end{array}$ \\
\hline Input & \begin{tabular}{|l}
$x_{00001}=00181998, x_{00011}=00181998$ \\
$x_{00101}=c 0 c c c 000, x_{00111}=c 0 c c c 000$ \\
$x_{01001}=0 c 0 c c c 00, x_{01011}=0 c 0 c c c 00$ \\
$x_{10100}=00 c 0 c 003, x_{10110}=00 c 0 c 003$ \\
$x_{11001}=0 c 0 c c c 00, x_{11011}=0 c 0 c c c 00$
\end{tabular} & $\frac{1}{4} \cdot \frac{1}{2}^{33}=2^{-35}$ & 76 \\
\hline 1 & $\mid \begin{array}{l}x_{00000}=06001806, x_{00010}=06001806, \\
x_{01101}=66600060, x_{01111}=66600060 \\
x_{10001}=06001806, x_{10011}=06001806, \\
x_{10101}=00 c 0 c 003, x_{10111}=00 c 0 c 003 \\
x_{11101}=60600180, x_{11111}=60600180\end{array}$ & $\left|\frac{1}{4} \cdot \frac{1}{2}^{39}=2^{-41}\right|$ & 64 \\
\hline 2 & $\mid \begin{array}{l}x_{00001}=06001806, x_{00011}=06001806 \\
x_{00101}=00 c 03030, x_{00111}=00 c 03030 \\
x_{01001}=000 c 0303, x_{01011}=000 c 0303 \\
x_{10100}=00000030, x_{10110}=00000030 \\
x_{11001}=000 c 0303, x_{11011}=000 c 0303\end{array}$ & $\left|\frac{1}{4} \cdot \frac{1}{2}^{17}=2^{-19}\right|$ & 52 \\
\hline 3 & $\mid \begin{array}{l}x_{00000}=00018000, x_{00010}=00018000 \\
x_{01101}=60181800, x_{01111}=60181800 \\
x_{10001}=00018000, x_{10011}=00018000 \\
x_{10101}=00000030, x_{10111}=00000030 \\
x_{11101}=00001800, x_{11111}=00001800\end{array}$ & $\frac{1}{4} \cdot \frac{1}{2}^{13}=2^{-15}$ & 28 \\
\hline 4 & $\begin{array}{l}x_{00001}=00018000, x_{00011}=00018000 \\
x_{00101}=0 c 000000, x_{00111}=0 c 000000 \\
x_{01001}=00 c 00000, x_{01011}=00 c 00000 \\
x_{11001}=00 c 00000, x_{11011}=00 c 00000\end{array}$ & $\frac{1}{4} \cdot \frac{1}{2}^{3}=2^{-5}$ & 16 \\
\hline 5 & $x_{01101}=00000006, x_{01111}=00000006$ & $\frac{1}{4} \cdot \frac{1}{2}^{1}=2^{-3}$ & 4 \\
\hline 6 & $x_{10100}=00000300, x_{10110}=00000300$ & $\frac{1}{4} \cdot \frac{1}{2}^{5}=2^{-7}$ & 4 \\
\hline 7 & $\mid \begin{array}{l}x_{00000}=00180000, x_{00010}=00180000 \\
x_{10001}=00180000, x_{10011}=00180000 \\
x_{10101}=00000300, x_{10111}=00000300 \\
x_{11101}=00018000, x_{11111}=00018000\end{array}$ & $\left|\frac{1}{4} \cdot \frac{1}{2}^{15}=2^{-17}\right|$ & 16 \\
\hline 8 & $\mid \begin{array}{l}x_{00001}=00180000, x_{00011}=00180000 \\
x_{00101}=c 0000000, x_{00111}=c 0000000 \\
x_{01001}=0 c 000000, x_{01011}=0 c 000000 \\
x_{10100}=c 0 c 00300, x_{10110}=c 0 c 00300 \\
x_{11001}=0 c 000000, x_{11011}=0 c 000000\end{array}$ & $\left|\frac{1}{4} \cdot \frac{1}{2}^{21}=2^{-23}\right|$ & 28 \\
\hline 9 & $\mid \begin{array}{l}x_{00000}=00180606, x_{00010}=00180606 \\
x_{01101}=00000060, x_{01111}=00000060 \\
x_{10001}=00180606, x_{10011}=00180606 \\
x_{10101}=c 0 c 00300, x_{10111}=c 0 c 00300 \\
x_{11101}=60018060, x_{11111}=60018060\end{array}$ & $\left|\frac{1}{4} \cdot \frac{1}{2}^{41}=2^{-43}\right|$ & 52 \\
\hline 10 & \begin{tabular}{|l}
$x_{00001}=00180606, x_{00011}=00180606$ \\
$x_{00101}=c 0303000, x_{00111}=c 0303000$ \\
$x_{01001}=0 c 030300, x_{01011}=0 c 030300$ \\
$x_{10100}=00303330, x_{10110}=00303330$ \\
$x_{11001}=0 c 030300, x_{11011}=0 c 030300$
\end{tabular} & $\frac{1}{4} \cdot \frac{1}{2}^{35}=2^{-37}$ & 64 \\
\hline 11 & $\mid \begin{array}{l}x_{00000}=81998001, x_{00010}=81998001 \\
x_{01101}=18180060, x_{01111}=18180060 \\
x_{10001}=81998001, x_{10011}=81998001 \\
x_{10101}=00303330, x_{10111}=00303330 \\
x_{11101}=18199800, x_{11111}=18199800\end{array}$ & & 76 \\
\hline
\end{tabular}

As the above approach sets many bits to zero (namely 33 bits to increase the bias by a factor 2), we offer a more efficient approach. One can fix only bits $i-1, i$ in $x_{0 j k \bar{l} m}$ and $i-1, i$ in $x_{1 j k \bar{l} m}$ to zero. Even if there is a carry entering bit $i-1$, it does not produce carry that affects the $i$ 'th bit, and we are assured that bit $i$ after the addition is indeed 0 . Therefore, to ensure that all the appropriate bits in $x_{1 j k l m}$ are 
Table 7. The 14-round linear approximation with bias $\frac{1}{4}^{\frac{1}{2}}{ }^{405}=2^{-407}$ rounds 1-9

\begin{tabular}{|c|c|c|c|}
\hline Round & $\begin{array}{c}\text { Mask } \\
\text { (before the round) }\end{array}$ & Bias & \begin{tabular}{|c|} 
Hamming \\
Weight
\end{tabular} \\
\hline input & $\begin{array}{l}x_{00001}=3 c c c f c 0 f, x_{00011}=3 c c c f c 0 f \\
x_{00101}=67 e 079 e 6, x_{00111}=67 e 079 e 6 \\
x_{01001}=667 e 079 e, x_{01011}=667 e 079 e \\
x_{10100}=66600060, x_{10110}=66600060 \\
x_{11001}=667 e 079 e, x_{11011}=667 e 079 e\end{array}$ & $\frac{1}{4} \cdot \frac{1}{2}^{60}=2^{-62}$ & 160 \\
\hline 1 & $\begin{array}{l}x_{00000}=00030333, x_{00010}=00030333, \\
x_{01101}=f 03 c f 333, x_{01111}=f 03 c f 333, \\
x_{10001}=00030333, x_{10011}=00030333, \\
x_{10101}=66600060, x_{10111}=66600060 \\
x_{11101}=30003033, x_{11111}=30003033\end{array}$ & $\frac{1}{4} \cdot \frac{1}{2}^{54}=2^{-56}$ & 100 \\
\hline 2 & $\begin{array}{l}x_{00001}=00030333, x_{00011}=00030333 \\
x_{00101}=18199800, x_{00111}=18199800 \\
x_{01001}=01819980, x_{01011}=01819980 \\
x_{10100}=60181800, x_{10110}=60181800 \\
x_{11001}=01819980, x_{11011}=01819980\end{array}$ & $\frac{1}{4} \cdot \frac{1}{2}^{34}=2^{-36}$ & 76 \\
\hline 3 & $\begin{array}{l}x_{00000}=c 0 c 00300, x_{00010}=c 0 c 00300 \\
x_{01101}=0 c c c 000 c, x_{01111}=0 c c c 000 c \\
x_{10001}=c 0 c 00300, x_{10011}=c 0 c 00300 \\
x_{10101}=60181800, x_{10111}=60181800 \\
x_{11101}=0 c 0 c 0030, x_{11111}=0 c 0 c 0030\end{array}$ & $\frac{1}{4} \cdot \frac{1}{2}^{40}=2^{-42}$ & 64 \\
\hline 4 & $\mid \begin{array}{l}x_{00001}=c 0 c 00300, x_{00011}=c 0 c 00300 \\
x_{00101}=00180606, x_{00111}=00180606 \\
x_{01001}=60018060, x_{01011}=60018060 \\
x_{10100}=00000006, x_{10110}=00000006 \\
x_{11001}=60018060, x_{11011}=60018060\end{array}$ & $\frac{1}{4} \cdot \frac{1}{2}^{18}=2^{-20}$ & 52 \\
\hline 5 & $\begin{array}{l}x_{00000}=00003000, x_{00010}=00003000 \\
x_{01101}=0 c 030300, x_{01111}=0 c 030300 \\
x_{10001}=00003000, x_{10011}=00003000 \\
x_{10101}=00000006, x_{10111}=00000006 \\
x_{11101}=00000300, x_{11111}=00000300\end{array}$ &,$\frac{1}{4} \cdot \frac{1}{2}^{14}=2^{-16}$ & 28 \\
\hline 6 & $\begin{array}{l}x_{00001}=00003000, x_{00011}=00003000 \\
x_{00101}=01800000, x_{00111}=01800000 \\
x_{01001}=00180000, x_{01011}=00180000 \\
x_{11001}=00180000, x_{11011}=00180000\end{array}$ & $\frac{1}{4} \cdot \frac{1}{2}^{4}=2^{-6}$ & 16 \\
\hline 7 & $x_{01101}=c 0000000, x_{01111}=c 0000000$ & 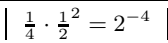 & 4 \\
\hline 8 & $x_{10100}=00000060, x_{10110}=00000060$ & $\frac{1}{4}_{4} \frac{1}{2}^{6}=2^{-8}$ & 4 \\
\hline 9 & $\begin{array}{l}x_{00000}=00030000, x_{00010}=00030000 \\
x_{10001}=00030000, x_{10011}=00030000 \\
x_{10101}=00000060, x_{10111}=00000060 \\
x_{11101}=00003000, x_{11111}=00003000\end{array}$ & $\frac{1}{4} \cdot \frac{1}{2}^{16}=2^{-18}$ & 16 \\
\hline
\end{tabular}

zero one needs to set the mask bits masked by $000 c 0 c c c_{x}$ of $x_{00001}, x_{00011}, x_{10001}$, and $x_{10011}$, the bits masked by $c 00 c 0001_{x}$ of $x_{00101}, x_{00111}, x_{10101}$, and $x_{10111}$, and those masked by $0 c 0 c c c 00$ in $x_{01000}, x_{01001}, x_{01010}, x_{01011}, x_{11000}, x_{11001}, x_{11010}$, and $x_{11011}$ to zero. Fixing these 116 bits (10 of which are shared with the previous 80 ), assures that all the additions in the first round of the 12-round approximation follow the approximation, i.e., "saving" their "contribution" to the bias, and resulting in a bias of $\frac{1}{4}^{2} \frac{1}{2}^{233}=2^{-235}$.

We note that the number of bits set to 0 is 186 , leaving 838 bits to be randomly selected. This is sufficient to generate the $2^{470}$ possible inputs to $T^{12}$, needed for using this chosen-plaintext linear approximation successfully, in a distinguishing attack on 12-round CubeHash. 
Table 8. The 14-round linear approximation with bias $\frac{1}{4} \cdot \frac{1}{2}^{405}=2^{-407}$ rounds $10-14$

\begin{tabular}{|c|c|c|c|}
\hline Round & $\begin{array}{c}\text { Mask } \\
\text { (before the round) }\end{array}$ & Bias & \begin{tabular}{|c} 
Hamming \\
Weight
\end{tabular} \\
\hline 10 & $\begin{array}{l}x_{00001}=00030000, x_{00011}=00030000, \\
x_{00101}=18000000, x_{00111}=18000000, \\
x_{01001}=01800000, x_{01011}=01800000, \\
x_{10100}=18180060, x_{10110}=18180060, \\
x_{11001}=01800000, x_{11011}=01800000\end{array}$ & $\frac{1}{4} \cdot \frac{1}{2}^{22}=2^{-24}$ & 28 \\
\hline 11 & $\mid \begin{array}{l}x_{00000}=c 00300 c 0, x_{00010}=c 00300 c 0, \\
x_{01101}=0000000 c, x_{0111}=0000000 c, \\
x_{10001}=c 00300 c 0, x_{10011}=c 00300 c 0, \\
x_{10101}=18180060, x_{10111}=18180060, \\
x_{11101}=0 c 00300 c, x_{1111}=0 c 00300 c\end{array}$ & $\left|\frac{1}{4} \cdot \frac{1}{2}^{42}=2^{-44}\right|$ & 52 \\
\hline 12 & $\begin{array}{l}x_{00001}=c 00300 c 0, x_{00011}=c 00300 c 0, \\
x_{00101}=18060600, x_{00111}=18060600, \\
x_{01001}=01806060, x_{01011}=01806060, \\
x_{10100}=00060666, x_{10110}=00060666, \\
x_{11001}=01806060, x_{11011}=01806060\end{array}$ & $\left|\frac{1}{4} \cdot \frac{1}{2}^{36}=2^{-38}\right|$ & 64 \\
\hline 13 & $\begin{array}{l}x_{00000}=30333000, x_{00010}=30333000, \\
x_{01101}=0303000 c, x_{01111}=0303000 c, \\
x_{10001}=30333000, x_{10011}=30333000, \\
x_{10101}=00060666, x_{10111}=00060666, \\
x_{11101}=03033300, x_{11111}=03033300\end{array}$ & $\frac{1}{4} \cdot \frac{1}{2}^{58}=2^{-60}$ & 76 \\
\hline 14 & $\begin{array}{l}x_{00001}=30333000, x_{00011}=30333000, \\
x_{00101}=99800181, x_{00111}=99800181, \\
x_{01001}=19980018, x_{01011}=19980018, \\
x_{10100}=999 f 81 e 7, x_{10110}=999 f 81 e 7, \\
x_{11001}=19980018, x_{11011}=19980018\end{array}$ & & 100 \\
\hline
\end{tabular}

Table 9. The round that extends the 11-round approximation to 12 rounds (and the bits to fix

\begin{tabular}{|c|c|c|}
\hline Round & Input mask & Input bits fixed to 0 \\
\hline-1 & $\begin{array}{l}x_{00000}=00181998, x_{00010}=00181998 \\
x_{01101}=81 e 7999 f, x_{01111}=81 e 7999 f \\
x_{10001}=00181998, x_{10011}=00181998 \\
x_{10101}=33000303, x_{10111}=33000303 \\
x_{11101}=80018199, x_{11111}=80018199\end{array}$ & $\begin{aligned} x_{10001}=00080888_{x}, x_{10011} & =00080888_{x} \\
x_{10101}=11000101_{x}, x_{10111} & =11000101_{x} \\
x_{11101}=f f f f f f f f_{x}, x_{1111} & =f f f f f f f f_{x}\end{aligned}$ \\
\hline-0.5 & $\begin{array}{c}x_{00101}=f 30 c 0300_{x}, x_{00111}=f 30 c 0300_{x} \\
x_{01000}=0 c 0 c c c 00_{x}, x_{01001}=0 c 0 c c c 00_{x} \\
x_{01010}=0 c 0 c c c 00_{x}, x_{01011}=0 c 0 c c c 00_{x} \\
x_{01101}=80018199_{x}, x_{01111}=80018199_{x} \\
x_{10001}=00181998_{x}, x_{10011}=00181998_{x} \\
x_{10101}=c 00 c 003, x_{10111}=c 00 c 003 \\
x_{11000}=0 c 0 c c c 00_{x}, x_{11001}=0 c 0 c c c 00_{x} \\
x_{11010}=0 c 0 c c c 00_{x}, x_{11011}=0 c 0 c c c 00_{x}\end{array}$ & $\begin{aligned} x_{00001}=000 c 0 c c c_{x}, x_{00011} & =000 c 0 c c c_{x} \\
x_{00101}=c 00 c 0001_{x}, x_{00111} & =c 00 c 0001_{x} \\
x_{01000}=0 c 0 c c c 00_{x}, x_{01001} & =0 c 0 c c c 00_{x} \\
x_{01010}=0 c 0 c c c 00_{x}, x_{01011} & =0 c 0 c c c 00_{x} \\
x_{10001}=000 c 0 c c c_{x}, x_{10011} & =000 c 0 c c c_{x} \\
x_{10101}=c 00 c 0001_{x}, x_{10111} & =c 00 c 0001_{x} \\
x_{11010}=0 c 0 c c c 00_{x}, x_{11011} & =0 c 0 c c c 00_{x} \\
x_{10001}=000 c 0 c c c_{x}, x_{10011} & =000 c 0 c c c_{x}\end{aligned}$ \\
\hline 0 & $\begin{aligned} x_{00001}=00181998, x_{00011} & =00181998 \\
x_{00101}=c 0 c c c 000, x_{00111} & =c 0 c c c 000 \\
x_{01001}=0 c 0 c c c 00, x_{01011} & =0 c 0 c c c 00 \\
x_{10100}=00 c 0 c 003, x_{10110} & =00 c 0 c 003 \\
x_{11001}=0 c 0 c c c 00, x_{11011} & =0 c 0 c c c 00\end{aligned}$ & \\
\hline
\end{tabular}

\section{Distinguishing Reduced-Round Variants of the Compression Function of CubeHash}

Given the linear approximations presented in the previous sections, it is possible to distinguish a black box which contains up to 12-round CubeHash from a random permutation. Of course, for any unkeyed primitive this distinguishing can be done 
by just comparing the input/output of a few queries to the black box with the input/output produced by the publicly available algorithm. If we want to offer some cryptographic settings in which distinguishing attacks make sense, we either need to consider keyed variants (either of the round function $T$ or of the hash function, e.g., in MACs) or to discuss known-key distinguishers [23].

Such possible "application" is an a Even-Mansour [15] variant of 11-round $T$ (or any other number of rounds), i.e., $E M-T_{k_{1}, k_{2}}^{11}(P)=T^{11}\left(P \oplus k_{1}\right) \oplus k_{2}$. If 11-round $T$ is indeed good as a source of nonlinearity (for a linear $T$, the entire security of CubeHash collapses), then XORing an unknown key before and after these 11 rounds, should result in a good pseudo-random permutation. Using our linear approximations, one can distinguish this construction from a random permutation.

We emphasize that as our results are linear in nature, they require that the adversary has access both to the input to the nonlinear function as well as its output. To the best of our knowledge, there is no way to use this directly in a hash function setting.

\section{Conclusions}

In this paper we presented a series of approximations for the SHA-3 candidate CubeHash. The analysis challenges the strength of CubeHash's round function, $T$, and shows that (from linear cryptanalysis point of view), offers adequate security. At the same time, the security margins offered by 16 iterations of $T$ seems to be on the smaller side, as future works on CubeHash may find better linear approximations.

\section{Acknowledgement}

The authors wish to thank Prof. Adi Shamir for his guidance and assistance analyzing CubeHash, Nathan Keller for providing core ideas in this paper, Daniel J. Bernstein for his insightful and mind-provoking comments on previous versions of this article. Finally, we wish to thank Michael Klots for his technical assistance, which was crucial for finding our results.

\section{References}

1. Andreeva, E., Bouillaguet, C., Fouque, P.A., Hoch, J.J., Kelsey, J., Shamir, A., Zimmer, S.: Second Preimage Attacks on Dithered Hash Functions. In Smart, N.P., ed.: EUROCRYPT. Volume 4965 of Lecture Notes in Computer Science., Springer (2008) 270-288

2. Aumasson, J.P.: Collision for CubeHash2/120-512. NIST mailing list (2008) Available online at http://ehash.iaik.tugraz.at/uploads/a/a9/Cubehash.txt.

3. Aumasson, J.P., Brier, E., Meier, W., Naya-Plasencia, M., Peyrin, T.: Inside the Hypercube. In Boyd, C., Nieto, J.M.G., eds.: ACISP. Volume 5594 of Lecture Notes in Computer Science., Springer (2009) 202-213

4. Bernstein, D.J.: CubeHash specification (2.B.1). Submission to NIST (2008)

5. Bernstein, D.J.: CubeHash specification (2.B.1). Submission to NIST (2009)

6. Biham, E., Chen, R.: Near-Collisions of SHA-0. [17] 290-305

7. Bloom, B., Kaminsky, A.: Single Block Attacks and Statistical Tests on CubeHash. IACR ePrint Archive, Report 2009/407 (2009)

8. Brier, E., Khazaei, S., Meier, W., Peyrin, T.: Linearization framework for collision attacks: Application to cubehash and md6. In Matsui, M., ed.: ASIACRYPT. Volume 5912 of Lecture Notes in Computer Science., Springer (2009) 560-577 
9. Brier, E., Khazaei, S., Meier, W., Peyrin, T.: Real Collisions for CubeHash-4/48. NIST mailing list (2009) Available online at http://ehash.iaik.tugraz.at/uploads/5/50/Bkmp_ch448.txt.

10. Brier, E., Khazaei, S., Meier, W., Peyrin, T.: Real Collisions for CubeHash-4/64. NIST mailing list (2009) Available online at http://ehash.iaik.tugraz.at/uploads/9/93/Bkmp_ch464.txt.

11. Cannière, C.D., Rechberger, C.: Finding SHA-1 Characteristics: General Results and Applications. In Lai, X., Chen, K., eds.: ASIACRYPT. Volume 4284 of Lecture Notes in Computer Science., Springer (2006) 1-20

12. Cho, J.Y., Pieprzyk, J.: Multiple Modular Additions and Crossword Puzzle Attack on NLSv2. In Garay, J.A., Lenstra, A.K., Mambo, M., Peralta, R., eds.: ISC. Volume 4779 of Lecture Notes in Computer Science., Springer (2007) 230-248

13. Cramer, R., ed.: Advances in Cryptology - EUROCRYPT 2005, 24th Annual International Conference on the Theory and Applications of Cryptographic Techniques, Aarhus, Denmark, May 22-26, 2005, Proceedings. In Cramer, R., ed.: EUROCRYPT. Volume 3494 of Lecture Notes in Computer Science., Springer (2005)

14. Dai, W.: Collisions for CubeHash1/45 and CubeHash2/89 (2008) Available online at http://www.cryptopp.com/sha3/cubehash.pdf.

15. Even, S., Mansour, Y.: A Construction of a Cipher from a Single Pseudorandom Permutation. J. Cryptology 10(3) (1997) 151-162

16. Ferguson, N., Lucks, S., McKay, K.A.: Symmetric States and their Structure: Improved Analysis of CubeHash. IACR ePrint Archive, Report 2010/273 (2010) Presented at the SHA-3 second workshop, Santa Barbara, USA, August 23-24, 2010.

17. Franklin, M.K., ed.: Advances in Cryptology - CRYPTO 2004, 24th Annual International CryptologyConference, Santa Barbara, California, USA, August 15-19, 2004, Proceedings. In Franklin, M.K., ed.: CRYPTO. Volume 3152 of Lecture Notes in Computer Science., Springer (2004)

18. Joux, A.: Multicollisions in Iterated Hash Functions. Application to Cascaded Constructions. [17] 306-316

19. Kelsey, J., Kohno, T.: Herding Hash Functions and the Nostradamus Attack. In Vaudenay, S., ed.: EUROCRYPT. Volume 4004 of Lecture Notes in Computer Science., Springer (2006) 183-200

20. Kelsey, J., Schneier, B.: Second Preimages on n-Bit Hash Functions for Much Less than $2^{\mathrm{n}}$ Work. [13] 474-490

21. Khovratovich, D., Nikolic', I., Weinmann, R.P.: Preimage attack on CubeHash512-r/4 and CubeHash512-r/8 (2008) Available online at http://ehash.iaik.tugraz.at/uploads/6/6c/Cubehash.pdf.

22. Knudsen, L.R., Mathiassen, J.E.: A Chosen-Plaintext Linear Attack on DES. In Schneier, B., ed.: FSE. Volume 1978 of Lecture Notes in Computer Science., Springer (2000) 262-272

23. Knudsen, L.R., Rijmen, V.: Known-Key Distinguishers for Some Block Ciphers. In Kurosawa, K., ed.: ASIACRYPT. Volume 4833 of Lecture Notes in Computer Science., Springer (2007) 315-324

24. Matsui, M.: Linear Cryptoanalysis Method for DES Cipher. In: EUROCRYPT. (1993) 386-397

25. National Institute of Standards and Technology: Cryptographic Hash Algorithm Competition. http://www.nist.gov/hash-competition (2008)

26. Stevens, M., Lenstra, A.K., de Weger, B.: Chosen-Prefix Collisions for MD5 and Colliding X.509 Certificates for Different Identities. In Naor, M., ed.: EUROCRYPT. Volume 4515 of Lecture Notes in Computer Science., Springer (2007) 1-22

27. Wang, X., Yu, H.: How to Break MD5 and Other Hash Functions. [13] 19-35 